\title{
Effects of formation properties in one-proton radioactivity
}

\author{
Chong Qi,, , $\rightarrow$ Doru S. Delion, ${ }^{2,3}$ Roberto J. Liotta, ${ }^{1}$ and Ramon Wyss ${ }^{1}$ \\ ${ }^{1} \mathrm{KTH}$, Alba Nova University Center, SE-10691 Stockholm, Sweden \\ 2 "Horia Hulubei" National Institute of Physics and Nuclear Engineering, \\ 407 Atomistilor, RO-07r125 Bucharest-Magurele, Romania \\ ${ }^{3}$ Academy of Romanian Scientists, 54 Splaiul Independentei, RO-050085 Bucharest, Romania
}

(Dated: September 6, 2018)

\begin{abstract}
It is shown that the proton formation probability, extracted from experimental data corresponding to one-proton radioactivity, is divided into two regions when plotted as a function of an universal parameter. This parameter is derived from a microscopic description of the decay process. In this way we explain the systematics of proton emission half-lives. At the same time the formation probability is shown to be a useful quantity to determine the deformation property of the mother nucleus.
\end{abstract}

PACS numbers: 23.50.+z, 21.30.Fe, 21.60.Gx, 21.10.Tg

Understanding how nuclear many-body systems can self-organize in simple and regular patterns is a longstanding challenge in modern physics. The first case where this was realized was in $\alpha$ radioactivity, where the regularity manifests itself as striking linear correlations between the logarithm of the decay half-life and the kinetic energy of the outgoing particle. This is known as the Geiger-Nuttall law, which was proposed 100 years ago [1]. This law has been so successful that even today it is applied in studies of radioactive decays (see, e.g., Refs. $[2,3])$. The reason for this success is that the $\alpha$-particle formation probability, which is neglected in the GeigerNuttall law, usually varies from nucleus to nucleus much less than the penetrability. In the logarithm scale of the Geiger-Nuttall law the differences in the formation probabilities are usually small fluctuations along the straight lines corresponding to the isotopic chains. The importance of a proper treatment of $\alpha$ decay was attested by a recent calculation which shows that the different lines can be merged in a single one [4]. The resulting universal decay law (UDL), which uses three free parameters only, explains well all known ground-state to ground-state radioactive decays. A similar three-term formula is also proposed in Refs. [5, [6].

This good agreement is a consequence of the smooth transition in the nuclear structure that is often found when going from a nucleus to its neighboring nuclei. This is also the reason why the BCS approximation works so well in many nuclear regions. Notable discrepancies are only seen in a few cases around the $N=126$ shell closure where clustering induced by the pairing mode is inhibited [7]. Nuclei can even undergo more complex heavy-cluster decays, but these also follow similar linear relations [4[6, 8,, 9$]$.

Besides these cluster decay cases, proton radioactivity also provides a unique opportunity to study the structure of nuclei close to the proton drip-line [10]. In the past decades tens of proton decay events have been observed in odd- $Z$ elements between $Z=53$ and $Z=83$, leading to an almost complete identification of the edge of nuclear stability in this region [11, 12]. On the theoretical side, one may extract from proton emission detailed information on the nuclear potential beyond the drip-line 13, 14] as well as the spectroscopic properties of the nuclei involved [15-17].

The proton-emission process can be looked as a quantum tunneling through the Coulomb and centrifugal barriers of a quasistationary state [18]. Similar to $\alpha$ and heavy cluster decays, the proton decay process can be divided into an "internal region", where the compound state is restricted, and the complementary "external region". This division is such that in the external region only the Coulomb and centrifugal forces are important and the decaying system behaves like a two-particle system. The corresponding expression for the half life can be written as 19]

$$
T_{1 / 2}=\frac{\hbar \ln 2}{\Gamma_{l}}=\frac{\ln 2}{\nu}\left|\frac{H_{l}^{+}(\chi, \rho)}{R \mathcal{F}_{l}(R)}\right|^{2},
$$

where $\nu$ and $l$ are the outgoing velocity and the angular momentum carried by the outgoing proton, respectively. $\mu$ is the reduced mass corresponding to the final system. $R$ is the radius dividing the internal and external regions. The half life does not depend upon $R$ [18]. At this point the wave function of the proton is matched with the corresponding outgoing wave function in the external region. In the following, the distance $R$ will be taken as the touching point, i.e., $R=R_{0}\left(A_{d}^{1 / 3}+1\right)$, with $R_{0}=1.2 \mathrm{fm}$. The other quantities are standard, i.e., $H_{l}^{+}(\chi, \rho)$ is the Coulomb-Hankel function with arguments $\chi=2 Z_{d} e^{2} / \hbar \nu$ and $\rho=\mu \nu R / \hbar$.

The amplitude of the wave function in the internal region is the formation amplitude,

$$
\mathcal{F}_{l}(R)=\int d \mathbf{R} d \xi_{d}\left[\Psi\left(\xi_{d}\right) \xi_{p} Y_{l}(\mathbf{R})\right]_{J_{m} M_{m}}^{*} \Psi_{m}\left(\xi_{d}, \xi_{p}, \mathbf{R}\right),
$$


TABLE I. Coefficient sets of Eq. (4) that determined by fitting to experiments of all proton decays (I) and to decays of nuclei with $N<75$ and $N \geq 75$ separately (II), and the corresponding rms deviations.

\begin{tabular}{ccccccc}
\hline \hline & Emitter & $\mathrm{a}$ & $\mathrm{b}$ & $\mathrm{c}$ & $\mathrm{d}$ & $\sigma$ \\
\hline I & all & 0.386 & -0.502 & 2.386 & -17.8 & 0.440 \\
& $N<75$ & 0.443 & -0.364 & 2.66 & -23.6 & 0.344 \\
II & $N<75$ & 0.403 & -0.110 & 2.766 & -27.8 & 0.235 \\
& $N \geq 0$ \\
\hline \hline
\end{tabular}

where $d, p$ and $m$ label the daughter, proton and mother nuclei, respectively. $\Psi$ are the intrinsic wave functions and $\xi$ the corresponding intrinsic coordinates. One sees from Eq. (2) that $\mathcal{F}_{l}(R)$ would indeed be the wave function of the outgoing particle $\psi_{p}(R)$ if the mother nucleus would behave at the point $R$ as

$$
\Psi_{m}\left(\xi_{d}, \xi_{p}, \mathbf{R}\right)=\left[\Psi\left(\xi_{d}\right) \xi_{p} \psi_{p}(R) Y_{l}(\mathbf{R})\right]_{J_{m} M_{m}} .
$$

By applying a similar technique as in Ref. [4] and considering the influence of the centrifugal barrier, one finds that the logarithm of the decay half-life can be approximated by

$$
\log T_{1 / 2}=a \chi^{\prime}+b \rho^{\prime}+d l(l+1) / \rho^{\prime}+c,
$$

where $a, b, c$ and $d$ are constants, $\chi^{\prime}=A^{1 / 2} Z_{p} Z_{d} Q_{p}^{-1 / 2}$, $\rho^{\prime}=\sqrt{A Z_{p} Z_{d}\left(A_{d}^{1 / 3}+A_{p}^{1 / 3}\right)}, A=A_{d} A_{p} /\left(A_{d}+A_{p}\right)$, and in this case of proton decay it is $Z_{p}=A_{p}=1$ (for details see Ref. [4]). Notice that $c$ depends on the formation amplitude $\mathcal{F}_{l}(R)$. This formula should be compared with that of Ref. 17] where a linear correlation between the reduced half-life and the Coulomb parameter was found.

The coefficients $a$ to $d$ can be determined by fitting available experimental data. For this purpose we took a total number of 44 decay events from the compilation of Ref. [12 and the recent results of Ref. 20]. The fitted values are listed in Table II It is seen that the log values of the experimental half-lives can be reproduced within an error of $\sigma=0.44$. Correspondingly, the decay halflives are reproduced within an average factor of three. This should be compared with the case of $\alpha$ decay where the UDL reproduces the available experimental half-lives within a factor of about 2.2 .

To probe Eq. (4), we plotted in the upper panel of Fig. 1 the quantity $\log T_{1 / 2}^{\text {Expt. }}-\left[b \rho^{\prime}+d l(l+1) / \rho^{\prime}+c\right]$ as a function of $\chi^{\prime}$. In the lower part of this Figure we plotted the discrepancy between experimental and calculated values, i.e., the ratio $\mathcal{R}=T_{1 / 2}^{\text {Expt. }} / T_{1 / 2}^{\text {Cal. }}$, as a function of the emitter charge numbers $Z$. The calculations were performed by using the parameter set I in Table I. It is seen that most of the data can be reproduced by the calculation within a factor of 4 , i.e., with $0.25 \leq \mathcal{R} \leq 4$. Larger discrepancies are seen for a emitters between $63 \leq Z \leq 67$ and the isomeric $h_{11 / 2}$ hole state in the $Z=81$ nucleus ${ }^{177} \mathrm{Tl}$, where the experimental decay half life is underestimated by the calculation by a factor of about 8 .

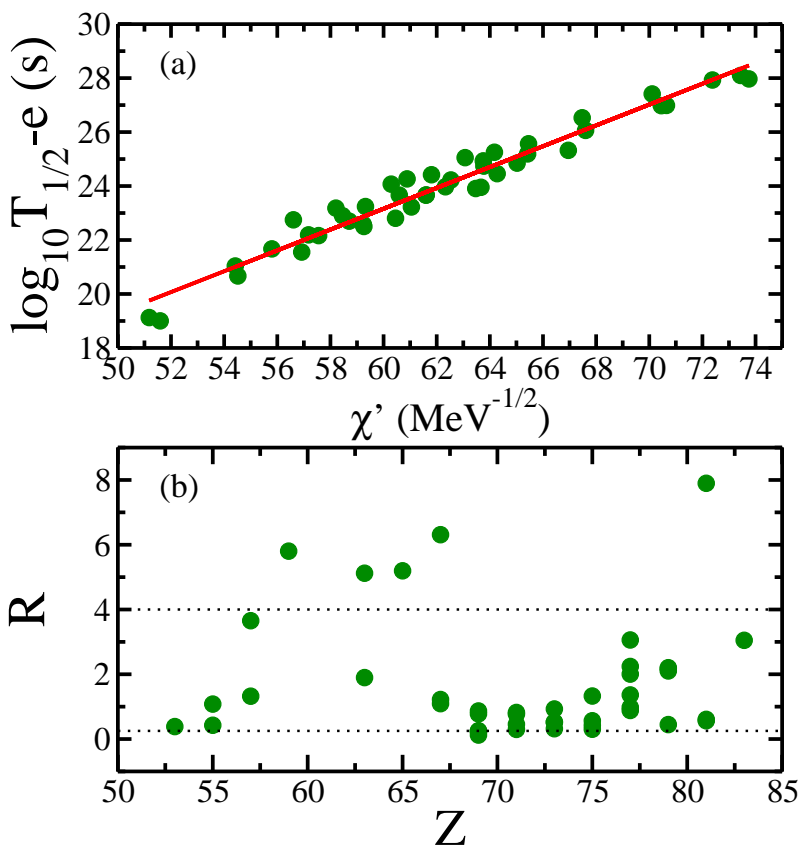

FIG. 1. (Color online) Upper: UDL proton decay half-lives. Dots are experimental values while the straight line is $a \chi^{\prime}$ with $a$ provided by the parameter set I of Table I. $e$ is given by $e=b \rho^{\prime}+d l(l+1) / \rho^{\prime}+c$. Lower: $\mathcal{R}=T_{1 / 2}^{\text {Expt. }} / T_{1 / 2}^{\text {Cal. }}$ as a function of the charge number $Z$. Within the dashed lines is the region where the data is reproduced by the calculation within a factor of 4 .

A better understanding of these discrepancies would require a systematic study of the formation probabilities. We performed this task by extracting the formation amplitudes $F(R)$ from the experimental half lives by using the expression [4],

$$
\log |R F(R)|^{-2}=\log T_{1 / 2}^{\text {Expt. }}-\log \left[\frac{\ln 2}{\nu}\left|H_{l}^{+}(\chi, \rho)\right|^{2}\right],
$$

which we plotted in Fig. 2 as a function of $\rho^{\prime}$.

One sees in this Figure that two clearly defined regions emerged, as indicated by the dashed lines which we obtained by using the parameter set II of Table [.

The origin of these two regions can be gathered from the values of the $Q$ values, deformation parameters and formation probabilities presented in Table III, where the experimental and corresponding theoretical half-lives are also given.

One sees in this Table that the region to the left in Fig. 2, i.e., for lighter isotopes, corresponds to the decays of well deformed nuclei. The formation probabilities decreases for these nuclei as $\rho^{\prime}$ increases. Then, suddenly, a strong transition occurs at $\rho^{\prime}=20.5$, corresponding to the nucleus ${ }_{69}^{144} \mathrm{Tm}$. Here the formation probability acquires its maximum value, and then decreases again as $\rho^{\prime}$ increases. One notices in Table II that this tendency is followed by the deformations, which gradually diminishes after the transition point. One thus sees that the 
TABLE II. Experimental and calculated half-lives for proton decays to ground states. Experimental $Q$ values and half-lives are taken from Refs. [12, 20]. Stars in the emitters indicate isomeric states. The deformation parameter $\beta_{2}$ are from Ref. 21]. The calculations were performed by using the parameter sets I $\left(T_{1 / 2}^{\text {Cal. }}(\mathrm{I})\right)$ and II $\left(T_{1 / 2}^{\text {Cal. }}\right.$ (II)) of Table I In the last two columns are the formation amplitudes $\left|F_{l}(R)\right|$ and formation probabilities $\left|R F_{l}(R)\right|^{2}$ (Eq. (15)). Units in the calculated half lives are as in the corresponding experimental values.

\begin{tabular}{|c|c|c|c|c|c|c|c|c|c|}
\hline Emitter & $l$ & $j_{m}^{\pi}$ & $Q(\mathrm{keV})$ & $\beta_{2}$ & $T_{1 / 2}^{\text {Expt. }}$ & $T_{1 / 2}^{\text {Cal. }}(\mathrm{I})$ & $T_{1 / 2}^{\text {Cal. }}(\mathrm{II})$ & $\left|F_{l}(R)\right|\left(\mathrm{fm}^{-3 / 2}\right)$ & $\left|R F_{l}(R)\right|^{2}\left(\mathrm{fm}^{-1}\right)$ \\
\hline${ }_{53}^{109} \mathrm{I}$ & 2 & $5 / 2^{+}$ & $827(5)$ & 0.160 & $93.5(5) \mu \mathrm{s}$ & 243 & 187 & 0.020 & 0.018 \\
\hline${ }_{55}^{112} \mathrm{Cs}$ & $(2)$ & $\left(0^{+}, 3^{+}\right)$ & $823(7)$ & 0.208 & $0.5(1) \mathrm{ms}$ & 1.18 & 1.39 & 0.021 & 0.021 \\
\hline${ }_{55}^{113} \mathrm{Cs}$ & 2 & $3 / 2^{+}$ & $976(3)$ & 0.207 & $16.7(7) \mu \mathrm{s}$ & 15.5 & 9.72 & 0.010 & 0.005 \\
\hline${ }_{57}^{117} \mathrm{La}$ & 2 & $3 / 2^{+}$ & $814(11)$ & 0.290 & $23.8(20) \mathrm{ms}$ & 6.52 & 12.3 & 0.008 & 0.003 \\
\hline${ }_{57}^{117} \mathrm{La}$ & 4 & $9 / 2^{+}$ & $951(6)$ & 0.290 & $10(5) \mathrm{ms}$ & 7.55 & 12.6 & 0.016 & 0.013 \\
\hline${ }_{59}^{121} \operatorname{Pr}$ & 2 & $3 / 2^{+}$ & $900(10)$ & 0.318 & $10\left({ }_{-3}^{+6}\right) \mathrm{ms}$ & 1.72 & 3.28 & 0.006 & 0.002 \\
\hline${ }_{63}^{130} \mathrm{Eu}$ & 2 & $1^{+}$ & $1039(15)$ & 0.331 & $0.90\left({ }_{-29}^{+49}\right) \mathrm{ms}$ & 0.474 & 1.12 & 0.010 & 0.005 \\
\hline${ }_{63}^{131} \mathrm{Eu}$ & 2 & $3 / 2^{+}$ & $959(9)$ & 0.331 & $21.4\left(\begin{array}{l}+1.8 \\
-1.7\end{array}\right) \mathrm{ms}$ & 4.18 & 13.8 & 0.007 & 0.002 \\
\hline${ }_{65}^{135} \mathrm{~Tb}$ & 3 & $7 / 2^{-}$ & $1200(7)$ & 0.325 & $0.94\left({ }_{-0.22}^{+0.33}\right) \mathrm{ms}$ & 0.181 & 0.428 & 0.006 & 0.002 \\
\hline${ }_{67}^{140} \mathrm{Ho}$ & 3 & $\left(6^{-}, 0^{-}, 8^{+}\right)$ & $1106(10)$ & 0.297 & $6(3) \mathrm{ms}$ & 4.91 & 23.3 & 0.016 & 0.014 \\
\hline${ }_{67}^{141} \mathrm{Ho}$ & 3 & $7 / 2^{-}$ & $1190(8)$ & 0.286 & $4.1(1) \mathrm{ms}$ & 0.650 & 2.30 & 0.006 & 0.002 \\
\hline${ }_{67}^{141} \mathrm{Ho}^{*}$ & 0 & $1 / 2^{+}$ & $1255(8)$ & 0.286 & $6.6\left({ }_{-0.7}^{+0.9}\right) \mu \mathrm{s}$ & 6.04 & 12.0 & 0.010 & 0.006 \\
\hline${ }_{69}^{144} \mathrm{Tm}$ & 5 & $\left(10^{+}, 5^{-}\right)$ & $1725(16)$ & 0.258 & $2.7\left(_{-0.7}^{+1.7}\right) \mu \mathrm{s}$ & 22.0 & 6.37 & 0.045 & 0.111 \\
\hline${ }_{69}^{145} \mathrm{Tm}$ & 5 & $11 / 2^{-}$ & $1753(7)$ & 0.249 & $\sim 3.46(32) \mu \mathrm{s}$ & 14.8 & 4.28 & 0.031 & 0.056 \\
\hline${ }_{69}^{146} \mathrm{Tm}$ & 5 & $\left(5^{-}\right)$ & $1210(4)$ & -0.199 & $117.6(64) \mathrm{ms}$ & 151 & 67.8 & 0.030 & 0.051 \\
\hline${ }_{69}^{146} \mathrm{Tm}^{*}$ & 5 & $\left(10^{+}\right)$ & $1140(4)$ & -0.199 & $203(6) \mathrm{ms}$ & 792 & 383 & 0.058 & 0.190 \\
\hline${ }_{69}^{147} \mathrm{Tm}$ & 5 & $11 / 2^{-}$ & $1073(5)$ & -0.190 & $3.78(1.27) \mathrm{s}$ & 4.36 & 2.32 & 0.035 & 0.069 \\
\hline${ }_{69}^{147} \mathrm{Tm}^{*}$ & 2 & $3 / 2^{+}$ & $1133(3)$ & -0.190 & $0.360(36) \mathrm{ms}$ & 1.51 & 0.269 & 0.031 & 0.056 \\
\hline${ }_{71}^{150} \mathrm{Lu}$ & 5 & $>5^{-}$ & $1283(3)$ & -0.164 & $64.0(56) \mathrm{ms}$ & 83.1 & 51.3 & 0.030 & 0.050 \\
\hline${ }_{71}^{150} \mathrm{Lu}^{*}$ & 2 & $\left(1^{-}, 2^{-}\right)$ & $1306(5)$ & -0.164 & $43\left({ }_{-5}^{+7}\right) \mu \mathrm{s}$ & 94.1 & 20.9 & 0.020 & 0.023 \\
\hline${ }_{71}^{151} \mathrm{Lu}$ & 5 & $11 / 2^{-}$ & $1253(3)$ & -0.156 & $127.1(18) \mathrm{ms}$ & 155 & 100 & 0.030 & 0.051 \\
\hline${ }_{71}^{151} \mathrm{Lu}^{*}$ & 2 & $3 / 2^{+}$ & $1332(10)$ & -0.156 & $16(1) \mu \mathrm{s}$ & 54.0 & 11.9 & 0.024 & 0.033 \\
\hline${ }_{73}^{155} \mathrm{Ta}$ & 5 & $11 / 2^{-}$ & $1468(15)$ & 0.008 & $2.9\left(\begin{array}{l}+1.5 \\
-1.1\end{array}\right) \mathrm{ms}$ & 5.56 & 4.37 & 0.031 & 0.056 \\
\hline${ }_{73}^{156} \mathrm{Ta}$ & 2 & $\left(2^{-}\right)$ & $1032(5)$ & -0.050 & $149(8) \mathrm{ms}$ & 286 & 135 & 0.029 & 0.050 \\
\hline${ }_{73}^{156} \mathrm{Ta}^{*}$ & 5 & $\left(9^{+}\right)$ & $1127(7)$ & -0.050 & $8.52(2.12) \mathrm{s}$ & 9.19 & 10.3 & 0.036 & 0.076 \\
\hline${ }_{73}^{157} \mathrm{Ta}$ & 0 & $1 / 2^{+}$ & $947(7)$ & 0.045 & $0.300(105) \mathrm{s}$ & 0.941 & 0.401 & 0.037 & 0.080 \\
\hline${ }_{75}^{159} \mathrm{Re}^{*}$ & 5 & $11 / 2^{-}$ & $1831(20)$ & 0.053 & $20.2(37) \mu \mathrm{s}$ & 47.7 & 42.6 & 0.026 & 0.041 \\
\hline${ }_{75}^{160} \mathrm{Re}$ & 2 & $\left(2^{-}\right)$ & $1287(6)$ & 0.080 & $0.687(11) \mathrm{ms}$ & 1.22 & 0.642 & 0.021 & 0.027 \\
\hline${ }_{75}^{161} \mathrm{Re}$ & 0 & $1 / 2^{+}$ & $1214(6)$ & 0.080 & $0.440(2) \mathrm{ms}$ & 1.45 & 0.660 & 0.027 & 0.045 \\
\hline${ }_{75}^{161} \mathrm{Re}^{*}$ & 5 & $11 / 2^{-}$ & $1338(6)$ & 0.080 & $224(31) \mathrm{ms}$ & 169 & 226 & 0.024 & 0.034 \\
\hline${ }_{77}^{164} \mathrm{Ir}$ & 5 & $\left(9^{+}\right)$ & $1844(9)$ & 0.089 & $0.113\left(\left(_{-30}^{+62}\right) \mathrm{ms}\right.$ & 0.0829 & 0.108 & 0.015 & 0.014 \\
\hline${ }_{77}^{165} \mathrm{Ir}^{*}$ & 5 & $11 / 2^{-}$ & $1733(7)$ & 0.099 & $0.34(7) \mathrm{ms}$ & 0.385 & 0.550 & 0.021 & 0.026 \\
\hline${ }_{77}^{166} \mathrm{Ir}$ & 2 & & $1168(7)$ & 0.107 & $0.152(71) \mathrm{s}$ & 0.0678 & 0.0631 & 0.014 & 0.011 \\
\hline${ }_{77}^{166} \mathrm{Ir}^{*}$ & 5 & $\left(9^{+}\right)$ & $1340(8)$ & 0.107 & $0.84(28) \mathrm{s}$ & 0.419 & 0.835 & 0.021 & 0.026 \\
\hline${ }_{77}^{167} \mathrm{Ir}$ & 0 & $1 / 2^{+}$ & 1096( 6) & 0.116 & $110(15) \mathrm{ms}$ & 112 & 0.919 & 0.020 & 0.023 \\
\hline${ }_{77}^{167} \operatorname{Ir}^{*}$ & 5 & $11 / 2^{-}$ & $1261(7)$ & 0.116 & $7.5(24) \mathrm{s}$ & 2.45 & 5.39 & 0.019 & 0.021 \\
\hline${ }_{79}^{170} \mathrm{Au}$ & 2 & $\left(2^{-}\right)$ & $1488(12)$ & 0.080 & $321\left({ }_{-58}^{+67}\right) \mu \mathrm{s}$ & 153 & 1.53 & 0.010 & 0.007 \\
\hline${ }_{79}^{170} \mathrm{Au}^{*}$ & 5 & $\left(9^{+}\right)$ & $1770(6)$ & 0.080 & $1.046\left({ }_{-126}^{+136}\right) \mathrm{ms}$ & 0.482 & 0.991 & 0.014 & 0.011 \\
\hline${ }_{79}^{171} \mathrm{Au}$ & 0 & $1 / 2^{+}$ & $1464(10)$ & -0.105 & $24.5\left(_{-3.1}^{+4.7}\right) \mu \mathrm{s}$ & 54.8 & 45.1 & 0.020 & 0.025 \\
\hline${ }_{79}^{171} \mathrm{Au}^{*}$ & 5 & $11 / 2^{-}$ & $1719(4)$ & -0.105 & $2.22(19) \mathrm{ms}$ & 1.01 & 2.18 & 0.014 & 0.012 \\
\hline${ }_{81}^{176} \mathrm{Tl}$ & 0 & $\left(3^{-}, 4^{-}, 5^{-}\right)$ & $1282(18)$ & -0.053 & $5.2\left({ }_{-1.4}^{+3.0}\right) \mathrm{ms}$ & 8.55 & 12.7 & 0.023 & 0.032 \\
\hline${ }_{81}^{177} \mathrm{Tl}$ & 0 & $1 / 2^{+}$ & $1180(20)$ & -0.053 & $67(37) \mathrm{ms}$ & 119 & 203 & 0.027 & 0.045 \\
\hline${ }_{81}^{177} \mathrm{Tl}^{*}$ & 5 & $11 / 2^{-}$ & $1984(8)$ & -0.053 & $396\left(_{-77}^{+87}\right) \mu \mathrm{s}$ & 50.1 & 137 & 0.006 & 0.003 \\
\hline${ }_{83}^{185} \mathrm{Bi}$ & 0 & $1 / 2^{+}$ & $1624(16)$ & -0.052 & $58(9) \mu \mathrm{s}$ & 19.0 & 33.1 & 0.008 & 0.004 \\
\hline
\end{tabular}




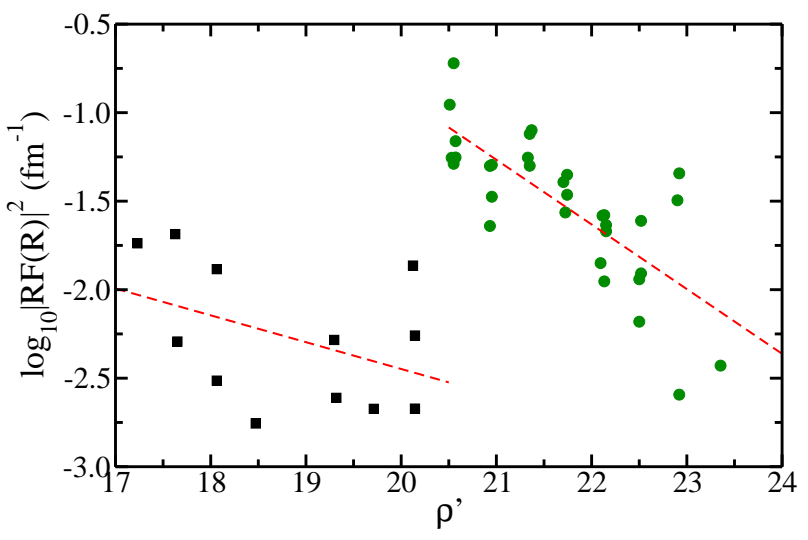

FIG. 2. (Color online) Proton-decay formation amplitudes $\log _{10}|R F(R)|^{2}$ extracted from experimental data as a function of $\rho^{\prime}$. Squares correspond to nuclei with $N<75(Z \leq 67)$ while circles are for $N \geq 75(Z>67)$.

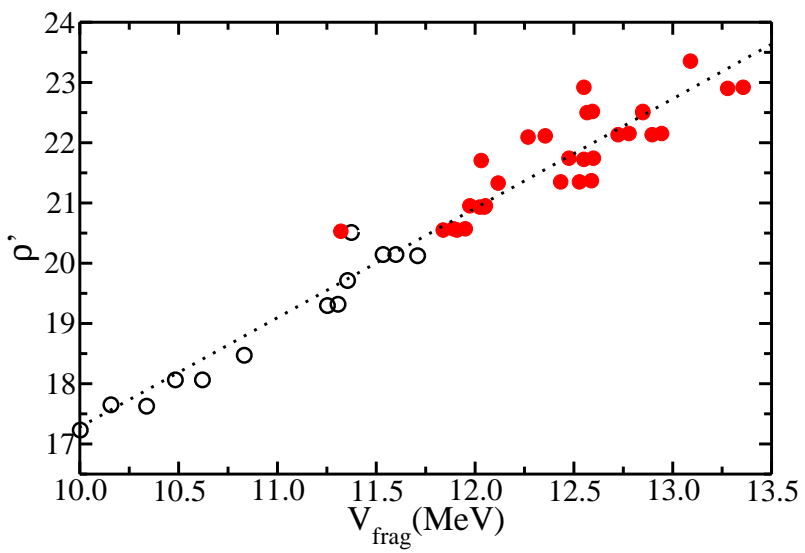

FIG. 3. The parameter $\rho^{\prime}$ versus the fragmentation potential (Eq. (6)) for nuclei with $Z \leq 67$ (open circles) and $Z>67$ (dark circles).

reason of this tendency of the formation amplitude is related to the deformation. Indeed, in the left region of Fig. 2, the decays of the deformed nuclei proceed through small spherical components of the corresponding deformed orbitals and, therefore, the formation probabilities are small.

The right region of Fig. 2 involves the decays of spherical orbits as well as major spherical components of deformed orbitals (for example, $h_{11 / 2}$ component of the orbital 11/2[505]).

Another striking feature is that the formation probability of the odd-odd nucleus ${ }^{144} \mathrm{Tm}$ is abnormally large. Considering that the experimental uncertainties regarding the half-life (from where the formation probability is extracted) is large one may doubt the validity of this anomaly. Moreover, it is not surprising, given this anomaly, that for ${ }^{144} \mathrm{Tm}$ the half-life evaluated with the set of parameters I differs from the corresponding experimental value by a factor $R$ which is beyond the scale used in the lower panel of Fig. 1]

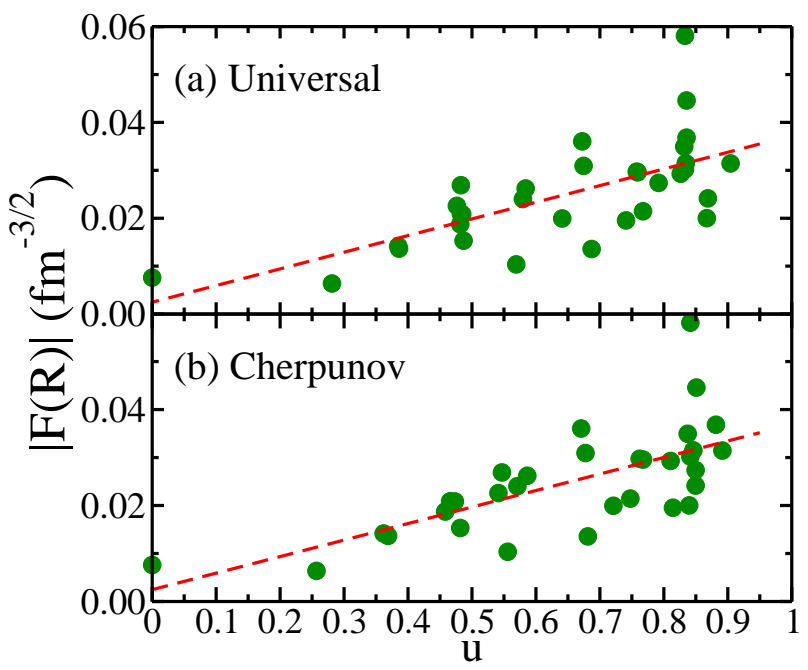

FIG. 4. (Color online) The formation amplitudes $\left|F_{l}(R)\right|$ extracted from experimental data for proton decays of nuclei $N \geq 75(Z>67)$ as a function of $u$ calculated from BCS calculations using for the Woods-Saxon mean field the universal parameters 23] (upper) and the Cherpunov parameters 24] (lower).

It is interesting to point out that a similar "clustering" of the proton wave functions into two distinct regions, as the one seen in Fig. 2, was evidenced in Ref. [22]. But there the determining variable was not $\rho^{\prime}$ but the so-called fragmentation potential. This is given by the difference between the Coulomb barrier and the $Q$-value as

$$
V_{\text {frag }}=\frac{Z_{d} e^{2}}{R}-Q .
$$

This feature is not surprising, since there is a linear correlation between these parameters, as can be seen in Fig. 3 where the parameter $\rho^{\prime}$ is plotted versus the above defined fragmentation potential. One sees here a clear linear correlation between these parameters. Moreover, the plot is divided into two regions with $Z \leq 67$ and $Z>67$. In fact that correlation is stronger than what the figure suggests since the upper part of the plot (dark circles) is divided into several regions where few close values of the fragmentation potential (with different $Q$ values) correspond to the same $\rho^{\prime}$. It is worthwhile to point out that the linear correlation between $V_{\text {frag }}$ and $\rho^{\prime}$, as seen in Fig. 3. is a result of the matching of the proton internal wave function with the corresponding external Coulomb wave. This is because both parameters are connected by this procedure.

Going back to Fig. 2 we notice that in the BCS approach the formation amplitude at a given radius $R$ would be proportional to the product of the occupancy $u$ times the single-proton wave function $\psi_{p}(R)$. Therefore the tendencies seen in Fig. 2 may be due to the BCS amplitudes or the radial wave functions. To recognize the influence of the value of the BCS amplitudes we plotted in 


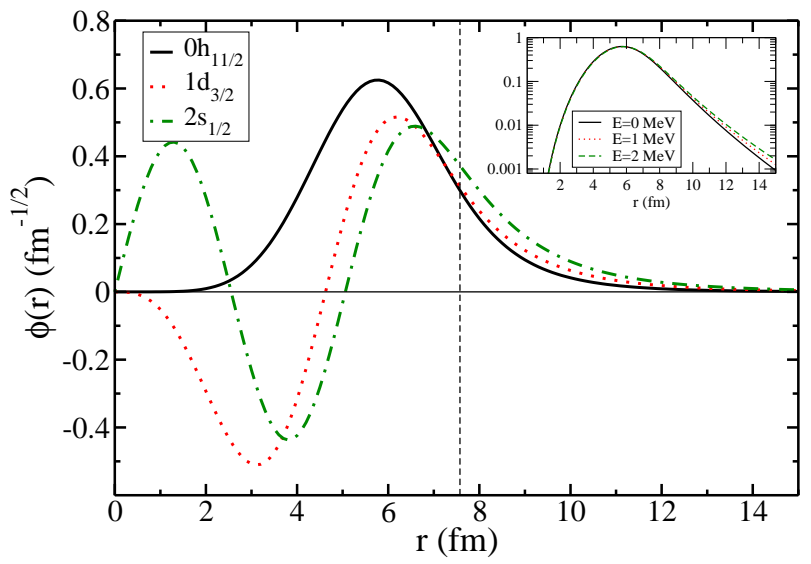

FIG. 5. (Color online) Single-proton wave functions in ${ }^{151} \mathrm{Lu}$ for different channels calculated with the universal WoodsSaxon parameter. The inserted plot shows the wave functions of the $0 h_{11 / 2}$ orbital with different energies derived by changing the depth of the potential.

Fig. 四 the formation probabilities $\left|F_{l}(R)\right|$ extracted from experiment for the case of proton decays corresponding to nuclei with $N \geq 75(Z>67)$ as a function of $u$. The $u$ values were calculated by using a Woods-Saxon potential according to the universal (upper) and Cherpunov (lower) parameters. One sees that the tendency is the same for both sets of parameters, namely that the values of of the formation probabilities increases with $u$, strengthening our interpretation of the behavior of the formation probabilities presented above.

Still one has to consider eventual changes in the radial wave functions at the nuclear radius (where the formation probabilities are determined). For this we plotted in Fig. 5 the single-particle wave functions $\phi(r)=r \psi_{p}(r)$ for different orbitals corresponding to the nucleus ${ }^{151} \mathrm{Lu}$. It is seen that the values of $\phi(r)$ around the nuclear surface are quite similar to each other. From this we conclude that the fluctuations in the experimental formation amplitudes found above for nuclei with $N \geq 75$ are mainly due to fluctuations of the $u$ values. It is also to be noticed that some cases which departs from the UDL correspond to the decays of hole states. This occurs in the isomeric state of ${ }^{177} \mathrm{Tl}$ (as already pointed out above) and also in the ground state of ${ }^{185} \mathrm{Bi}[25]$.
Another feature that has to be considered is the influence (if any) of the $Q$ value upon the formation probabilities. The $Q$ value determines the penetrability and, therefore, the radioactive decay process. The question is whether even the spectroscopic quantities are affected by the $Q$ value. In Ref. [22] it was evidenced the fact that the logarithm of the wave function is proportional to the fragmentation potential (Eq. (6)) for all decay processes, in particular for proton emission. This is a direct consequence of the one-body Schrödinger equation. Anyway, it seems that the dependence on the $Q$-value alone is small in proton decay processes. To analyze this we show in the inserted plot of Fig. 5 the wave functions of the $0 h_{11 / 2}$ orbital under different energies. These were obtained by changing the depth of the potential. As perhaps expected, the formation amplitude at the nuclear surface is not sensitive to changes in the energy, i.e., in the $Q$ values. Neither the amplitudes $u$ are much affected by the changing of the potential depth, as also expected. One therefore concludes that the changes in the formation amplitudes extracted from experimental data are a result of changes in the deformation of the decaying nuclei.

In conclusion, we have shown in this paper that the proton formation probability in the corresponding decay process depends upon the deformation of the decaying nucleus. In a well deformed nucleus the decay proceeds through one of the spherical components of the deformed orbit, which is usually small in this case of large deformations. Therefore the formation probability is small. On the contrary, in spherical or weakly deformed nuclei the decay proceeds through the only component that is available and, as a result, the formation probability is large. We have strengthen this interpretation by showing that in the case of weakly deformed nuclei the formation probability is proportional to the BCS occupation number $u$ corresponding to the decaying orbit.

This work was supported by the Swedish Research Council (VR) under grant Nos. 623-2009-7340 and 6212010-4723 and by a grant of the Romanian National Authority for Scientific Research, CNCS-UEFISCDI, project number PN-II-ID-PCE-2011-3-0092. CQ also acknowledges the computational support provided by the Swedish National Infrastructure for Computing (SNIC) at PDC and NSC.
[1] H. Geiger and J.M. Nuttall, Philos. Mag. 22, 613 (1911); H. Geiger, Z. Phys. 8, 45 (1922).

[2] G. Royer, Nucl. Phys. A 848, 279 (2010).

[3] V. I. Zagrebaev, A. V. Karpov, I. N. Mishustin, and W. Greiner, Phys. Rev. C 84, 044617 (2011).

[4] C. Qi, F. R. Xu, R. J. Liotta, and R. Wyss, Phys. Rev. Lett. 103, 072501 (2009); Phys. Rev. C 80, 044326 (2009).

[5] Z. Ren, C. Xu, and Z. Wang, Phys. Rev. C 70, 034304 (2004).
[6] D. Ni, Z. Ren, T. Dong, and C. Xu, Phys. Rev. C 78, 044310 (2008).

[7] C. Qi, A.N. Andreyev, M. Huyse, R.J. Liotta, P. Van Duppen, R.A. Wyss, Phys. Rev. C 81, 064319 (2010).

[8] D. N. Poenaru, R. A. Gherghescu, and W. Greiner, Phys. Rev. Lett. 107, 062503 (2011).

[9] D. N. Poenaru, R. A. Gherghescu, and W. Greiner, Phys. Rev. C 83, 014601 (2011).

[10] S. Hofmann, in Particle Emission from Nuclei, edited by D. N. Poenaru and M. S. Ivascu (CRC Press, Boca 
Raton, 1989), Vol. II, p. 25.

[11] P.J. Woods and C.N. Davids, Ann. Rev. Nucl. Part. Sci. 47, 541 (1997).

[12] B. Blank, M.J.G. Borge, Prog. Part. Nucl. Phys. 60, 403 (2008).

[13] B. Buck, A.C. Merchant and S.M. Perez, Phys. Rev. C 45, 1688 (1992).

[14] L.S. Ferreira, E. Maglione, and D.E.P. Fernandes, Phys. Rev. C 65, 024323 (2002).

[15] S. Åberg, P.B. Semmes and W. Nazarewicz, Phys. Rev. C 56, 1762 (1997).

[16] C.N. Davids et al., Phys. Rev. Lett. 76, 592 (1996).

[17] D.S. Delion, R.J. Liotta, and R. Wyss, Phys. Rev. Lett.
96, 072501 (2006).

[18] D.S. Delion, R.J. Liotta, and R. Wyss, Phys. Rep. 424, 113 (2006).

[19] D.S. Delion, Theory of Particle and Cluster Emission, Lecture Notes in Physics 819, Springer, 2010.

[20] I. G. Darby et al., Phys. Rev. C 83, 064320 (2011).

[21] P. Möller, R.J. Nix, W.D. Myers, and W. Swiatecki, At. Data Nucl. Data Tables 59, 185 (1995).

[22] D.S. Delion, Phys. Rev. C 80, 024310 (2009).

[23] S. Cwiok, J. Dudek and W. Nazarewicz, Comput. Phys. Commun. 46379 (1987).

[24] V.A. Cherpunov, Yad. Fiz. 6, 955 (1967).

[25] G. L. Poli et al., Phys. Rev. C 59, R2979 (1999). 